\title{
Improving survival and preventing recurrence of diffuse large B-cell lymphoma in younger patients: current strategies and future directions
}

This article was published in the following Dove Press journal:

OncoTargets and Therapy

29 March 2013

Number of times this article has been viewed

\author{
Lionel Karlin \\ Bertrand Coiffier \\ Hematology Department, Centre \\ Hospitalier Lyon Sud, Pierre-Benite, \\ France
}

\begin{abstract}
Prognosis of diffuse large B-cell lymphoma (DLBCL) has considerably improved during the last decade, mainly due to the addition of rituximab to chemotherapy. However, a significant proportion of patients still experience primary refractory disease or short-term relapses, conferring poor survival. Thus, achieving first-line complete remission is of major importance, especially in young and fit patients. Current strategies are based on the age-adapted International Prognostic Index, which separates patients into three prognostic subgroups (lowrisk, intermediate-risk, and high-risk). However, it is based only on clinical variables, and we have learned from daily practice that there remains a marked heterogeneity within each subgroup. Recently, biological prognostic factors have emerged, and should now be part of initial evaluation to guide treatment. Among those, so-called double-hit DLBCL with deregulation of both MYC and BCL2 genes usually follows a particularly aggressive course and should be treated more intensively. But for many other patients, the indication of high-dose therapy rather than immunochemotherapy alone remains controversial. In these cases, the interest of an early ${ }^{18} \mathrm{~F}$ fluoro-2-deoxy-D-glucose positron emission tomography evaluation-based strategy is now being assessed in ongoing clinical trials. Moreover, other strategies to improve response and survival consist in adding novel agents to standard chemotherapy. In this field, newly developed anti-CD20 monoclonal antibodies and immunomodulatory drugs could be of particular interest during induction therapy to optimize the quality of response, but also in maintenance treatment, in order to decrease the risk of relapse. Only well-conducted clinical trials will be able to resolve all these issues. Therefore, physicians should be encouraged, as far as possible, to propose them to their patients.
\end{abstract}

Keywords: diffuse large B-cell lymphoma, risk-adapted therapy, prognostic markers, early FDG-PET, novel agents, targeted therapy

\section{Introduction}

Diffuse large B-cell lymphoma (DLBCL) is the most common non-Hodgkin's lymphoma (NHL), accounting for approximately $30 \%$ of cases. ${ }^{1}$ During the last decade, prognosis of DLBCL considerably improved, mostly due to the addition of rituximab (R), the first approved anti-CD20 monoclonal antibody, to the CHOP regimen ${ }^{2}$ (Table 1). In the French Groupe d'Etudes des Lymphomes de l'Adulte (GELA) pivotal phase III trial, elderly patients ( $>60$ years of age) diagnosed with DLBCL and treated with an R-CHOP combination demonstrated a significantly higher complete response $(\mathrm{CR})$ rate $(76 \% \mathrm{vs}$ $63 \%$ ) and 2-year overall survival (OS) than patients receiving CHOP alone. Long-term analysis at 5- and 10-year follow-up then confirmed the survival advantage found in the R-CHOP arm., Concordant results were published by other cooperative groups.
Correspondence: Bertrand Coiffier

Hematology Department,

Centre Hospitalier Lyon Sud,

165 Chemin du Grand Revoyet,

Pierre-Bénite 69495, France

Tel +3347886430 I

Fax +33478864355

Email bertrand.coiffier@univ-lyonl.fr 
Table I Summary of the main studies on role of addition of rituximab to standard first-line therapy in DLBCL

\begin{tabular}{|c|c|c|c|c|}
\hline Study & Age (years) & Chemotherapy & Median follow-up & Results \\
\hline \multirow[t]{4}{*}{ Coiffier et a $\left.\right|^{2}$} & $60-80$ & $\mathrm{R}-\mathrm{CHOP} \times 8$ & 10 years & 10-year PFS: \\
\hline & & vs $\mathrm{CHOP} \times 8$ & & $36.5 \%$ vs $20 \% *$ \\
\hline & & & & 10-year OS: \\
\hline & & & & $43 \%$ vs $27 \% *$ \\
\hline \multirow[t]{4}{*}{ Pfreundschuh et $\mathrm{al}^{7}$} & $18-60$ & R-CHOP-like $\times 6$ & 72 months & 6-year PFS $=80.2 \%$ \\
\hline & & vs $\mathrm{CHOP}$-like $\times 6$ & & vs $63.9 \% *$ \\
\hline & & & & 6 -year OS $=90 \%$ \\
\hline & & & & vs $80 \% *$ \\
\hline \multirow[t]{5}{*}{ Habermann et al ${ }^{5}$} & $>60$ & Ist random R-CHOP $\times 6$ & 3.5 years & After induction alone: \\
\hline & & vs $\mathrm{CHOP} \times 6$ & & 3 -year FFS = \\
\hline & & 2nd random: $\mathrm{R}$ maintenance & & $53 \%$ vs $46 \% *$ \\
\hline & & vs observation & & 3 -year OS $=67 \%$ \\
\hline & & & & vs $58 \% *$ \\
\hline
\end{tabular}

Note: *Statistically significant.

Abbreviations: PFS, progression-free survival; FFS, failure-free survival; OS, overall survival.

In the German RICOVER-60 trial, six cycles of R-CHOP14 followed by two cycles of rituximab significantly improved event-free survival (EFS) and OS compared to six cycles of CHOP14 alone. ${ }^{5}$ An American study also showed in elderly DLBCL patients significantly better failure-free survival and OS rates in patients assigned to an R-CHOP regimen. ${ }^{6}$ Importantly, the benefit of rituximab added to $\mathrm{CHOP}$ was also established in young DLBCL patients. The MInT study randomized 824 newly diagnosed DLBCL patients aged from 18 to 60 years to either six cycles of CHOP or R-CHOP. Similarly, the $\mathrm{CR}$ rate was found significantly higher in patients assigned to R-CHOP, translating after a median follow-up of 34 months into better EFS and OS. ${ }^{7}$ This was confirmed by the long-term results after 6 years' follow-up. ${ }^{8}$

However, despite all these improvements in first-line treatment, there are still approximately $40 \%$ of patients who will experience either refractoriness or short-time relapses, mainly occurring in the first 2-3 following years. Moreover, such early relapses are often chemoresistant, leading to significantly shorter survival. Accordingly, and contrary to low-grade lymphomas, the objective of induction therapy must be cure, which seems mandatory for long-term disease-free survival.

After focusing on current strategies in the treatment of DLBCL in younger patients, this review will develop the following issues:

- how to improve CR rate by opting for new strategies consisting either in the addition of new agents to standard chemotherapy or in more intensive schemes of treatment

- whether there are new prognostic factors, more accurate than existing scoring, able to clearly discriminate patients at low risk from patients at high risk of relapse, for a well-defined risk-adapted therapy

- whether there is a place for postinduction maintenance treatment in order to reduce the risk of early relapse.

\section{Current strategy in young DLBCL patients}

So far, current recommendations have mainly been stratified on the age-adapted International Prognostic Index (aaIPI) score, which should be systematically calculated before treatment is initiated (Table 2). ${ }^{9}$ This score is based on the three following independent variables:

- stage according to Ann Arbor scale (I-II vs III-IV)

- performance status (0-1 vs 2-4)

- lactate dehydrogenase levels (normal vs elevated).

An aaIPI score of 0 defines low-risk patients, while a score of 1 or 2 defines intermediate-risk patients (respectively, low-intermediate and high-intermediate). Highrisk patients are represented by patients having aaIPI score of 3 .

\section{Low-risk patients}

In low-risk and nonbulky patients (aaIPI $=0$ ), the classically offered therapy consists of six cycles of R-CHOP delivered at 3-week intervals (R-CHOP 21). Ongoing trials are testing the noninferiority of four cycles of R-CHOP 21.

Table 2 Current strategies based on aalPI score in newly diagnosed young DLBCL patients

\begin{tabular}{lll}
\hline aalPI score & Risk & Strategy \\
\hline 0 & Low & R-CHOP $\times 6$ \\
I & Low-intermediate & R-ACVBP $\times 4$ \\
& & Sequential consolidation \\
2 & High-intermediate & R-ACVBP $\times 4$ HDT-ASCT \\
3 & High & R-ACVBP $\times 4$ HDT-ASCT \\
\hline
\end{tabular}

Notes: aalPI PS $\geq 2$ vs 0-I; Stage III-IV vs I-II; Elevated LDH vs normal. Abbreviations: R-CHOP, rituximab, cyclophosphamide, doxorubicin, vincristine, and prednisone; R-ACVBP, rituximab, doxorubicin, cyclophosphamide, bleomycin, and prednisone; HDT: high-dose therapy; ASCT: autologous stem cell transplant. 
In the currently recruiting LYSA trial, LNH-091B, lowrisk patients are randomized to six cycles of R-CHOP 21 (standard arm), or four cycles of R-CHOP 21 (experimental arm) for patients having a negative PET after the first two cycles.

\section{Low-intermediate-risk patients}

Treatment of low-intermediate-risk patients (aaIPI $=1)$ is traditionally based on eight cycles of R-CHOP 21 . However, some researchers have raised the possibility of dose-intensity approaches in the aim of improving initial response and optimizing the rate of cure. In the eighties, the GELA investigators developed the so-called ACVBP regimen, combining doxorubicin and cyclophosphamide (at a higher dose than in the CHOP regimen), vindesine, bleomycin, and prednisone. In the rituximab era, the LNH03-2B trial confirmed the superiority of this regimen over standard R-CHOP. This multicenter open-label randomized trial compared four cycles of R-ACVBP followed by sequential consolidation (consisting of two cycles of high-dose methotrexate, four cycles of rituximab combined with ifosfamide and etoposide, and finally two cycles of subcutaneous aracytine) to a standard R-CHOP regimen in low-intermediate-risk DLBCL patients. ${ }^{10}$ Three hundred eighty patients aged 18-59 years were included. There was no difference in terms of overall response $(90 \%$ vs $87 \%)$ and CR + unconfirmed CR (CRu) (83\% vs $80 \%$ ) between the two arms, but in an intent-to-treat analysis, 3-year progression-free survival (PFS) and OS were significantly longer in the R-ACVBP arm ( $87 \%$ vs $73 \%, P=0.015 ; 92 \%$ vs $84 \%, P=0.0071$, respectively). Grade 3-4 cytopenias and febrile neutropenias were more common in the experimental arm, but life-threatening complications were rare.

\section{High-intermediate and high-risk patients (aalPI $\geq 2$ )}

The $\mathrm{CR} / \mathrm{CRu}$ rate in this category of patient remains disappointing, and does not exceed $65 \%$ in the majority of the studies. In those patients, the role of high-dose therapy followed by autologous stem cell transplantation (HDT-ASCT) is still a matter of debate. Controversial results have been reported, but most of these trials were conducted in the pre-rituximab era. However, some studies suggested that high-intermediate and high-risk DLBCL patients could benefit from HDT-ASCT after a rituximab-containing induction regimen. Thus, in the LNH2003-3 phase II study conducted by GELA, 209 patients under 60 years received an induction phase with four cycles of R-ACVP followed by HDT-ASCT. ${ }^{11}$ A matched-pair analysis with patients treated with ACVBP and HDT-ASCT in the LNH1998-3 trial showed an increase of 4 years' OS after the addition of rituximab (74\% vs 58\%). In addition, the increase of CR + CRu from $60 \%$ after induction to $73 \%$ after ASCT also suggests a role of the intensive treatment and not only of rituximab in the improvement of response. However, other studies from the French GOELAMS study group and also from the GITIL Italian study group (the latest presented at the last American Society of Hematology [ASH] meeting) failed to show a superiority of HDT-ASCT over a dose-intensive rituximabcontaining regimen alone. ${ }^{12,13}$ Accordingly, there is still a need to clearly discriminate patients who will necessitate the use of HDT-ASCT from patients who will be cured with immunochemotherapy alone. This is currently approached with the use of early PET evaluation, in the context of clinical trials (see below). Pending these results, in our institution, an R-ACVBP induction scheme followed by HDT-ASCT is offered to aaIPI $2-3$ patients.

\section{Identification of new prognostic markers}

As previously described, management of DLBCL patients is guided by aaIPI score, which is to date the best available clinical tool to risk-stratify them. However, within each prognostic subgroup, there remains a marked heterogeneity in clinical outcomes, suggesting the existence of biological parameters that are probably not taken into account by this clinical-only variables-based index. In particular, it does not identify those patients who will follow an especially aggressive course, and who consecutively will have a markedly reduced survival. Therefore, in recent years, many efforts have been made to find biological prognostic markers able to identify such aggressive forms.

In this field, gene-expression profiling (GEP) studies have been conducted to divide DLBCL into two major molecular subtypes, based on cell-of-origin gene signatures: germinal center B-cell (GCB) type and activated B-cell $(\mathrm{ABC})$ type, the latter being associated with inferior OS. ${ }^{14}$ However, to date, GEP has not been routinely feasible. Therefore, an attempt was made to correlate each of these two subtypes with immunohistochemical (IHC) markers, which are CD10 and BCL6 for GCB type, and MUM-1/ IRF-4 for ABC type. But so far, such an algorithm has not demonstrated clear prognostic relevance and is not commonly used to guide therapy.

In an attempt to go further in the study of the prognostic significance of such IHC markers, especially in the rituximab era, the Lunenburg Lymphoma Biomarker 
Consortium evaluated - using tissue microarrays issued from 1514 patients included in twelve prospective clinical studies from European and American collaborative groups - the prognostic value of the following IHC markers: BCL2, BCL6, CD5, CD10, MUM1, Ki67, and HLA-DR. ${ }^{15}$ In patients treated with immunochemotherapy, only CD5 expression and $\mathrm{Ki} 67$ level appeared to be of prognostic value for OS, whereas the GCB/non-GCB IHC-based algorithm was not discriminant. At the end of the day, aaIPI seems to remain the best available prognostic index to date, meaning that molecular markers have still to be identified.

Based on the importance of alterations in oncogenes and tumor-suppressor genes in the pathogenesis of DLBCL, deregulation of the $M Y C$ and $B L C 2$ genes were recently suggested as poor prognosis factors. This deregulation can result either from chromosomal translocation or from gene amplification. In more detail, the combination of the alteration of these two oncogenes, the former promoting cell proliferation and the latter inhibiting apoptosis, and defining double-hit DLBCL seems to confer to the disease a particularly grim natural history. The impact of such a combination of genetic abnormalities was established in two recently published studies. In the first, Green et al performed fluorescence in situ hybridization (FISH) analysis and immunohistochemistry on biopsies of 193 de novo DLBCL patients who were uniformly treated with R-CHOP. ${ }^{16}$ Simultaneous rearrangement of the two genes was found in $6 \%$ of patients, conferring a significantly shorter median OS as compared to non-double-hit patients (13 vs 95 months, respectively), whereas translocation of one of these two oncogenes was not found to impact survival. FISH analysis being unable to detect MYC deregulation from other mechanisms than translocation, the measure of MYC and BCL2 expression by immunohistochemistry was performed. MYC was considered overexpressed when detected in more than $40 \%$ of lymphoma cells, and BCL2 when detected in more than 70\% of lymphoma cells, defining the "double-hit score" (DHS). Patients with no hyperexpression of one of these markers were considered DHS-0, whereas those with overexpression of one marker were considered DHS-1, and those with overexpression of the two markers were considered DHS-2, the latter representing $29 \%$ of patients. By univariate analysis, DHS-2 patients demonstrated significantly inferior PFS and OS compared to DHS-0/-1 patients, independently of GCB or ABC subtype, and also independently of aaIPI. Of note, high expression of MYC or BCL2 alone was not of prognostic significance. Similar results were found by Johnson et al, after measuring by an immunohistochemical method the concurrent expression of MYC and BCL2 in 167 DLBCL patients who received R-CHOP therapy. ${ }^{17}$ Here again, only coexpression of the two oncogenes, found in $21 \%$ of patients, was significantly associated with poorer PFS and OS, also independently of IPI score or molecular subtype. Taken together, these data call for a systematic assessment of BCL2 and MYC expression by gene analysis and immunohistochemical analysis. So-called double-hit patients thus could benefit from more intensive treatment or novel therapies, even if no standard of care is yet defined for those types of lymphoma. This is particularly underlined by the fact that their survival doesn't seem to be improved by intensive chemotherapy-based salvage treatment. ${ }^{18}$

Finally, very recently, results from an international consortium program study on a large cohort of DLBCL patients treated with R-CHOP found TP53 mutations to have strong prognostic value for survival, independently of GEP subgroups. ${ }^{19}$

\section{Early FDG-PET evaluation-based therapy}

Given the importance, in terms of clinical outcome, of obtaining CR after first-line induction in DLBCL patients, computed tomography-based end-of-treatment evaluation remains unsatisfactory. Indeed, in the frequent occurrence of a residual mass, such anatomic imaging is not able to discriminate active disease from fibrosis. The emergence of functional nuclear imaging and particularly of ${ }^{18} \mathrm{~F}$ fluoro-2deoxy-D-glucose positron emission tomography (FDG-PET) provided more accurate tools regarding prognosis after completion of treatment, the achievement of metabolic CR being correlated with survival. ${ }^{20}$ However, such delayed information cannot be used to guide treatment strategies. Thus, the prognostic value of interim FDG-PET, after one to four cycles of chemotherapy, has been addressed in several studies, all showing correlation between negative early FDG-PET and survival. However, most of them were performed before the rituximab era. Moreover, the use of a visual qualitative approach has been associated with falsepositive results.

Thus, Safar et al retrospectively studied a series of 112 newly diagnosed DLBCL patients treated with R-CHOP or R-ACVBP and in whom PET-FDG was performed after two cycles of treatment. ${ }^{21}$ Interpretation of PET-FDG was performed according to both qualitative (= visual) and quantitative methods. According to the qualitative method, negativity is defined by the absence of any abnormal uptake. The quantitative method is based on the degree of reduction 
of standardized uptake value $\left(\Delta \mathrm{SUV}_{\max }\right)$ between initial and interim PET, the cutoff for negativity being a $\triangle \mathrm{SUV}_{\text {max }}$ greater than $66 \%$. Patients achieving PET negativity after two cycles of therapy demonstrated significant improvement in 3 -year PFS (84\% vs $47 \%, P<0.0001)$ and OS (88\% vs $62 \%, P=0.003$ ) compared to patients who did not. This PFS advantage was also found by using the quantitative approach (77\% vs 38\%, $P=0.002)$.

Accordingly, sequential use of PET-FDG, in contrast to aaIPI, introduces a dynamic marker, probably better reflecting the natural evolution of lymphoma and therefore more in accordance with biology of the tumor. Interim PET-FDG results could be used to guide the modalities of continuation of therapy in a risk-adapted treatment approach. It means that patients obtaining early PET negativity could be spared from a more intensive approach, with the aim of limiting toxicity, and conversely patients remaining PET-positive could be offered HDT-ASCT. However, there has not been any randomized study showing that modifying the treatment on interim PET results has changed the outcome of the patients.

Two ongoing LYSA group trials are currently studying such modalities. In the LNH-091b trial, patients with newly diagnosed aaIPI $=0$ DLBCL are randomized either to six cycles of R-CHOP (standard treatment) or four cycles only in case of PET negativity after two cycles of therapy (experimental arm). Similarly, but in intermediate- or high-risk young patients, the recently opened GAINED trial proposes an early FDG-PET-driven strategy. All patients are evaluated by FDG-PET after two (PET2) and four (PET4) cycles of therapy by a quantitative method. Patients having negative PET2 and negative PET4, considered as early good responders, will receive immunochemotherapy-based sequential consolidation, while patients having positive PET2 and negative PET4, considered as late responders, will receive HDT-ASCT.

Finally, in a recently published study, Miyazaki et al retrospectively analyzed 50 de novo DLBCL patients and found a correlation between initial high $\mathrm{SUV}_{\max }$ and shorter PFS. $^{22}$

\section{New agents in first-line treatment of DLBCL}

Despite a dramatic reduction in the rate of relapse and failure in DLBCL in the last decade, the proportion of patients not achieving CR or relapsing shortly after first-line therapy remains significant. Therefore, there is a need for more effective first-line treatments, especially in high-risk patients. Among the current studies focusing on new agents, particular attention has been dedicated to new monoclonal antibodies, and also to immunomodulatory drugs.

\section{Monoclonal antibodies}

Regarding previous publications dealing with the interest of adding rituximab to chemotherapy and as a common cell antigen expressed on B cells, CD20 remains the most suitable antibody target for the treatment of DLBCL. Thus, recent efforts in the field of anti CD20 monoclonal antibodies have been made to improve their efficacy: on the one hand, by increasing direct cell-death capacities, through modifications of variable regions; on the other hand, by increasing immune effector functions such as antibody-dependent cytotoxicity (ADCC), through intrinsic changes of the Fc region of the antibody. In this field of investigation, Obinutuzumab (GA-101) was designed as a third-generation, humanized, and glycoengineered anti-CD20 antibody developed from the parental murine antibody B-ly1. ${ }^{23}$ It exhibits typical characteristics of a type II anti-CD20 antibody, with a lower complement-dependent cytotoxicity but a much superior cell death than rituximab. ${ }^{24}$ In addition, GA101 also harbors a glycoengineered, afucosylated Fc segment, resulting in a much more important binding affinity for Fc $\gamma$ RIIIa than rituximab, translating into an increased induction of ADCC. ${ }^{25}$ Thus, GA-101 could be more effective than rituximab. Although studies regarding GA-101 mainly focused on indolent lymphoma, some showed clinical activity in relapsing aggressive NHL. Cartron et al reported a phase II study in which seven of $25(28 \%)$ relapsed DLBCL achieved tumor response. ${ }^{26}$

In addition to the question of interim FDG-PETdriven strategy, the aforementioned GAINED trial also offers patients the choice of being randomized between R-chemotherapy or GA-101-chemotherapy. This trial was recently opened to inclusions.

Finally, newly designed antibody-drug conjugates have shown interesting results, which were presented during the last ASH meeting. ${ }^{27,28}$

\section{Immunomodulatory drugs}

Lenalidomide, an immunomodulatory drug derived from thalidomide, and with proven activity in multiple myeloma and chronic lymphocytic leukemia, has recently received increasing interest in NHL. ${ }^{29,30}$ The mechanism of action results from immunomodulatory properties, enhancing NK-cell antitumoral activity and altering the balance between pro- and anti-inflammatory cytokines leading to increased apoptosis, and also from antiangiogenic properties. ${ }^{30,31}$ The first phase II study of single-agent lenalidomide in relapsed/refractory NHL 
was performed on 49 patients, including 53\% with DLBCL histology. The overall response rate (ORR) in this subgroup was $19 \%$, with an acceptable toxicity, the most common grade 3-4 adverse events being cytopenias, generally easily manageable. Neither flare nor tumor lysis syndrome was observed. ${ }^{32}$ Another phase II study was conducted on 217 relapsed/ refractory patients, including $108 \mathrm{DLBCL}$, with an ORR of $28 \%{ }^{33}$ Based on these results and on the synergistic in vitro activity observed in combination with rituximab, a phase I study combining R-CHOP + lenalidomide (R2CHOP) was performed on 20 newly diagnosed DLBCL patients. ${ }^{34}$ Lenalidomide dose-escalation levels were $15 \mathrm{mg}, 20 \mathrm{mg}$, and $25 \mathrm{mg}$, administered on days $1-10$. No dose-limiting toxicity was found, and a dose of $25 \mathrm{mg}$ on days 1-10 was recommended for the corresponding ongoing phase II study. The most grade 3-4 adverse events were hematological (neutropenia and thrombocytopenia), but febrile neutropenia rarely occurred. The ORR was $100 \%$, with a CR rate of $77 \%$. Confirming these promising results, another phase II study, presented at the 2012 ASH meeting, was conducted on 49 DLBCL patients who received CHOP-R plus lenalidomide $15 \mathrm{mg}$ days 1-14. ORR was $92 \%$, with $86 \%$ CR. After a median follow-up of 18 months, OS was $94 \%$ and PFS 75\%. ${ }^{35}$ Interestingly, recently published data showed a better sensitivity to lenalidomide in non-GCB than in GCB relapsed/refractory patients. ${ }^{36}$

The previously described immunomodulatory properties of lenalidomide, combined with a good tolerability profile and an oral formulation, make it a good candidate for maintenance treatment. Such use has been now widely studied in multiple myeloma, and phase III studies are currently ongoing in chronic lymphocytic leukemia. So far, there have been no data on maintenance treatment of lenalidomide in DLBCL. Reddy and colleagues conducted a phase II randomized trial of lenalidomide alone or in combination with rituximab as maintenance treatment in CR-achieving DLBCL patients. The respective 2-year disease-free survival rates of $100 \%$ and $92 \%$ compared favorably with the approximate $70 \%$ diseasefree survival of historical cohorts. ${ }^{37}$ The ongoing LNH 09-6B (REMARC) trial (NCT01122472) conducted by LYSA cooperative group has also applied this strategy to newly diagnosed DLBCL patients aged 60-80 years. Responders after R-CHOP therapy are randomized to lenalidomide maintenance $(25 \mathrm{mg} /$ day, days $1-21)$ or placebo in the following 2 years, known as the period where most relapses occur.

\section{Other drugs}

Various new agents are currently being tested as part of early phase clinical trials, with an increasing role of targeted therapies. Several specific signaling pathways have emerged as important contributors to pathogenesis, leading to the development of new (and often oral) drugs. Numerous PI3 K-AKT pathway inhibitors are now evaluated. Promising results have also been found with the so-called PCI-32765, acting as Bruton's tyrosine kinase inhibitor. ${ }^{38}$ In this field, notably interesting results issued from a phase II study were communicated at the last $\mathrm{ASH}$ meeting, although the efficacy of the compound seems to be limited to the ABC subtype. ${ }^{39}$

Most of these agents are at this time used as single drugs in relapsed/refractory patients. However, there is a rationale to combine them with standard immunochemotherapy with the aim of improving ORR after first-line therapy.

\section{Conclusion}

Prognosis of DLBCL has considerably improved during the past 10 years. However, not all patients achieve cure of their disease, and the treatment of relapses remains unsatisfactory. Therefore, reaching CR after first-line therapy is an essential goal, but the benefit of intensive treatments must be balanced with the risk of toxicity, especially in young patients. Identification of new biological markers, routinely applicable, and early FDG-PET evaluation will help to improve such risk-adapted therapy. Moreover, recent data regarding the addition of novel agents to standard induction chemotherapy are particularly promising, especially in high-risk patients. Through its immunomodulatory properties, lenalidomide is of major interest in maintenance therapy, with the aim of reducing the risk of relapse. Only well-conducted clinical trials will be able to resolve all these issues. We encourage physicians, as far as possible, to refer their patients to reference centers participating in such trials.

\section{Disclosure}

Bertrand Coiffier: Celgene, honoraria; Roche, consultancy, honoraria. Lionel Karlin: Celgene, honoraria, expert board committee; Janssen, honoraria.

\section{References}

1. Coiffier B. State-of-the-art therapeutics: diffuse large B-cell lymphoma. J Clin Oncol. 2005 Sep 10;23(26):6387-93.

2. Coiffier B, Lepage E, Briere J, et al. CHOP chemotherapy plus rituximab compared with CHOP alone in elderly patients with diffuse large-B-cell lymphoma. N Engl J Med. 2002;346(4):235-242.

3. Coiffier B, Thieblemont C, Van Den Neste E, et al. Long-term outcome of patients in the LNH-98.5 trial, the first randomized study comparing rituximab-CHOP to standard CHOP chemotherapy in DLBCL patients: a study by the Groupe d'Etudes des Lymphomes de l'Adulte. Blood. 2010;116(12):2040-2045. 
4. Feugier P, Van Hoof A, Sebban C, et al. Long-term results of the R-CHOP study in the treatment of elderly patients with diffuse large B-cell lymphoma: a study by the Groupe d'Etude des Lymphomes de l'Adulte. J Clin Oncol. 2005;23(18):4117-4126.

5. Pfreundschuh M, Schubert J, Ziepert M, et al. Six versus eight cycles of bi-weekly CHOP-14 with or without rituximab in elderly patients with aggressive CD20+ B-cell lymphomas: a randomised controlled trial (RICOVER-60). Lancet Oncol. 2008;9(2):105-116.

6. Habermann TM, Weller EA, Morrison VA, et al. Rituximab-CHOP versus CHOP alone or with maintenance rituximab in older patients with diffuse large B-cell lymphoma. J Clin Oncol. 2006;24(19): 3121-3127.

7. Pfreundschuh M, Trumper L, Osterborg A, et al. CHOP-like chemotherapy plus rituximab versus CHOP-like chemotherapy alone in young patients with good-prognosis diffuse large-B-cell lymphoma: a randomised controlled trial by the MabThera International Trial (MInT) Group. Lancet Oncol. 2006;7(5):379-391.

8. Pfreundschuh M, Kuhnt E, Trumper L, et al. CHOP-like chemotherapy with or without rituximab in young patients with good-prognosis diffuse large-B-cell lymphoma: 6-year results of an open-label randomised study of the MabThera International Trial (MInT) Group. Lancet Oncol. 2011;12(11):1013-1022.

9. Tilly H, Dreyling M. Diffuse large B-cell non-Hodgkin's lymphoma: ESMO Clinical Practice Guidelines for diagnosis, treatment and followup. Ann Oncol. 2010;21 Suppl 5:v172-v174.

10. Recher C, Coiffier B, Haioun C, et al. Intensified chemotherapy with ACVBP plus rituximab versus standard $\mathrm{CHOP}$ plus rituximab for the treatment of diffuse large B-cell lymphoma (LNH03-02B): an open-label randomised phase 3 trial. Lancet. 2011;378(9806): 1858-1867.

11. Fitoussi O, Belhadj K, Mounier N, et al. Survival impact of rituximab combined with ACVBP and upfront consolidation autotransplantation in high-risk diffuse large B-cell lymphoma for GELA. Haematologica. 2011;96(8):1136-1143.

12. Cortelazzo S, Tarella C, Gianni A. Chemoimmunotherapy with R-CHOP or high dose sequential therapy with ASCT for high-risk DLBCL patients: results of the randomized R-HDS0305 trial by Gruppo Italiano Terapie Innovative Nei Linfomi (GITIL). 54th ASH Annual Meeting and Exposition; December 8-11, 2012; Atlanta, GA, USA.

13. Le Gouill S, Milpied N, Lamy T. First-line rituximab high-dose therapy versus R-CHOP14 for young adults with diffuse large B-cell lymphoma: Preliminary results of the GOELAMS 075 prospective multicenter randomized trial. J Clin Oncol. 2011;29 Suppl:8003.

14. Rosenwald A, Wright G, Chan WC, et al. The use of molecular profiling to predict survival after chemotherapy for diffuse large-B-cell lymphoma. N Engl J Med. 2002;346(25):1937-1947.

15. Salles G, de Jong D, Xie W, et al. Prognostic significance of immunohistochemical biomarkers in diffuse large B-cell lymphoma: a study from the Lunenburg Lymphoma Biomarker Consortium. Blood 2011;117(26):7070-7078.

16. Green TM, Young KH, Visco C, et al. Immunohistochemical doublehit score is a strong predictor of outcome in patients with diffuse large B-cell lymphoma treated with rituximab plus cyclophosphamide, doxorubicin, vincristine, and prednisone. J Clin Oncol. 2012;30(28): 3460-3467.

17. Johnson NA, Slack GW, Savage KJ, et al. Concurrent expression of MYC and BCL2 in diffuse large B-cell lymphoma treated with rituximab plus cyclophosphamide, doxorubicin, vincristine, and prednisone. J Clin Oncol. 2012;30(28):3452-3459.

18. Cuccuini W, Briere J, Mounier N, et al. MYC+ diffuse large B-cell lymphoma is not salvaged by classical R-ICE or R-DHAP followed by BEAM plus autologous stem cell transplantation. Blood. 2012;119(20): 4619-4624.

19. Xu-Monette ZY, Wu L, Visco C, et al. Mutational profile and prognostic significance of TP53 in diffuse large B-cell lymphoma patients treated with R-CHOP: report from an International DLBCL Rituximab-CHOP Consortium Program Study. Blood. 2012;120(19):3986-3996.
20. Jerusalem G, Beguin Y, Fassotte MF, et al. Persistent tumor 18F-FDG uptake after a few cycles of polychemotherapy is predictive of treatment failure in non-Hodgkin's lymphoma. Haematologica. 2000;85(6): 613-618.

21. Safar V, Dupuis J, Itti E, et al. Interim [18F]fluorodeoxyglucose positron emission tomography scan in diffuse large B-cell lymphoma treated with anthracycline-based chemotherapy plus rituximab. J Clin Oncol. 2012;30(2):184-190.

22. Miyazaki Y, Nawa Y, Miyagawa M, et al. Maximum standard uptake value of (18)F-fluorodeoxyglucose positron emission tomography is a prognostic factor for progression-free survival of newly diagnosed patients with diffuse large B cell lymphoma. Ann Hematol. 2013;92(2): 239-244.

23. Mössner E, Brünker P, Moser S, et al. Increasing the efficacy of CD20 antibody therapy through the engineering of a new type II anti-CD20 antibody with enhanced direct and immune effector cell-mediated B-cell cytotoxicity. Blood. 2010;115(22):4393-4402.

24. Robak T. GA-101, a third-generation, humanized and glyco-engineered anti-CD20 mAb for the treatment of B-cell lymphoid malignancies. Curr Opin Investig Drugs. 2009;10(6):588-596.

25. Ferrara C, Brünker P, Suter T, Moser S, Püntener U, Umaña P. Modulation of therapeutic antibody effector functions by glycosylation engineering: influence of Golgi enzyme localization domain and coexpression of heterologous beta1, 4-N-acetylglucosaminyltransferase III and Golgi alpha-mannosidase II. Biotechnol Bioeng. 2006;93(5): $851-861$.

26. Cartron G, Thieblemont C, Solal-Celigny P. Results from a phase II study of RO5072759 (GA101) monotherapy in relapsed refractory aggressive non-Hodgkin's lymphoma. Ann Oncol. 2011; 22:068.

27. Advani R, Lebovic D, Brunvand M. A phase I study of DCDT2980S, an antibody-drug conjugate targeting CD22, in relapsed or refractory B-cell NHL. 54th ASH Annual Meeting and Exposition; December 8-11, 2012; Atlanta, GA, USA.

28. Palanca-Wessels M, Flinn I, Sehn LH. A phase I study of the anti-CD79b antibody-drug conjugate DCDS4501 A targeting CD79b in relapsed or refractory B-cell NHL. 54th ASH Annual Meeting and Exposition; December 8-11, 2012; Atlanta, GA, USA.

29. Gunnellini M, Falchi L. Therapeutic activity of lenalidomide in mantle cell lymphoma and indolent non-Hodgkin's lymphomas. Adv Hematol. 2012;2012:523842.

30. Mounier N, Gisselbrecht C. Relapses, treatments and new drugs. Best Pract Res Clin Haematol. 2012;25(1):49-60.

31. Dawar R, Hernandez-Ilizaliturri F. The emerging role of lenalidomide in the management of mantle cell lymphoma (MCL). Best Pract Res Clin Haematol. 2012;25(2):185-190.

32. Wiernik PH, Lossos IS, Tuscano JM, et al. Lenalidomide monotherapy in relapsed or refractory aggressive non-Hodgkin's lymphoma. J Clin Oncol. 2008;26(30):4952-4957.

33. Witzig TE, Vose JM, Zinzani PL, et al. An international phase II trial of single-agent lenalidomide for relapsed or refractory aggressive B-cell non-Hodgkin's lymphoma. Ann Oncol. 2011;22(7): $1622-1627$.

34. Nowakowski GS, LaPlant B, Habermann TM, et al. Lenalidomide can be safely combined with R-CHOP (R2CHOP) in the initial chemotherapy for aggressive B-cell lymphomas: phase I study. Leukemia. 2011;25(12): 1877-1881.

35. Chiapella A, Franceschetti S, Castellino A. Rituximab-CHOP21 plus lenalidomide (LR-CHOP21) is effective and feasible in elderly untreated diffuse large B-cell lymphoma (DLBCL): results of phase II REAL07 study of the Fondazione Italiana Linfomi (FIL). 54th ASH Annual Meeting and Exposition; December 8-11, 2012; Atlanta, GA, USA.

36. Hernandez-Ilizaliturri FJ, Deeb G, Zinzani PL, et al. Higher response to lenalidomide in relapsed/refractory diffuse large B-cell lymphoma in nongerminal center B-cell-like than in germinal center B-cell-like phenotype. Cancer. 2011;117(22):5058-5066. 
37. Reddy NM, Simmons R, Caldwell M. A phase II randomized study of lenalidomide or lenalidomide and Rituximab as maintenance therapy following R-CHOP chemotherapy for patients with high-risk diffuse large B-cell lymphoma. 54th ASH Annual Meeting and Exposition; December 8-11, 2012; Atlanta, GA, USA.

38. Advani R, Sharman J, Smith S. The btk inhibitor pci-32765 is highly active and well tolerated in patients with relapsed/refractory B cell malignancies: final results from a phase I study. Ann Oncol. 2011;22:144.
39. Wilson WH, Gerecitano J, Goy A. The Bruton's tyrosine kinase inhibitor, ibrutinib (PCI-32765), has preferential activity in the ABC subtype of relapsed/refractory de novo DLBCL: interim results of a multicenter, open-label, phase 2 study. 54th ASH Annual Meeting and Exposition; December 8-11, 2012; Atlanta, GA, USA.

\section{Publish your work in this journal}

OncoTargets and Therapy is an international, peer-reviewed, open access journal focusing on the pathological basis of all cancers, potential targets for therapy and treatment protocols employed to improve the management of cancer patients. The journal also focuses on the impact of management programs and new therapeutic agents and protocols on
Dovepress

patient perspectives such as quality of life, adherence and satisfaction The manuscript management system is completely online and includes a very quick and fair peer-review system, which is all easy to use. Visit http://www.dovepress.com/testimonials.php to read real quotes from published authors.

\footnotetext{
Submit your manuscript here: http://www.dovepress.com/oncotargets-and-therapy-journal
} 